\title{
Protein arginine methyltransferase 3 -induced metabolic reprogramming is a vulnerable target of pancreatic cancer
}

\author{
Ming-Chuan Hsu', Ya-Li Tsai ${ }^{1}$, Chia-Hsien Lin ${ }^{1}$, Mei-Ren Pan², Yan-Shen Shan ${ }^{3,4}$, Tsung-Yen Cheng ${ }^{5}$, \\ Skye Hung-Chun Cheng ${ }^{6}$, Li-Tzong Chen ${ }^{1,7,8}$ and Wen-Chun Hung ${ }^{1,8^{*}}$
}

\begin{abstract}
Background: The biological function of protein arginine methyltransferase 3 (PRMT3) is not well known because very few physiological substrates of this methyltransferase have been identified to date.

Methods: The clinical significance of PRMT3 in pancreatic cancer was studied by database analysis. The PRMT3 protein level of human pancreatic tumors was detected by immunoblotting and immunohistochemical staining. PRMT3-associated proteins and the methylation sites on the proteins were investigated using mass spectrometry. Seahorse Bioscience analyzed the metabolic reprogramming. Combination index analysis and xenograft animal model were conducted to explore the effects of combination of inhibitors of glyceraldehyde-3-phosphate dehydrogenase (GAPDH) and oxidative phosphorylation on tumor growth.

Results: We found that the expression of PRMT3 is upregulated in pancreatic cancer, and its expression is associated with poor survival. We identified GAPDH as a PRMT3-binding protein and demonstrated that GAPDH is methylated at R248 by PRMT3 in vivo. The methylation of GAPDH by PRMT3 enhanced its catalytic activity while the mutation of R248 abolished the effect. In cells, PRMT3 overexpression triggered metabolic reprogramming and enhanced glycolysis and mitochondrial respiration simultaneously in a GAPDH-dependent manner. PRMT3-overexpressing cancer cells were addicted to GAPDH-mediated metabolism and sensitive to the inhibition of GAPDH and mitochondrial respiration. The combination of inhibitors of GAPDH and oxidative phosphorylation induced a synergistic inhibition on cellular growth in vitro and in vivo.
\end{abstract}

Conclusion: Our results suggest that PRMT3 mediates metabolic reprogramming and cellular proliferation through methylating R248 of GAPDH, and double blockade of GAPDH and mitochondrial respiration could be a novel strategy for the treatment of PRMT3-overexpressing pancreatic cancer.

Keywords: Protein arginine methyltransferase 3, Methylation, Glyceraldehyde-3-phosphate dehydrogenase, Metabolic reprogramming

\section{Background}

The methylation of arginine residues in cellular proteins by protein arginine methyltransferases (PRMTs) is an important posttranslational modification that modulates diverse cellular processes including gene transcription, DNA repair, messenger RNA processing, and signal

\footnotetext{
* Correspondence: hung1228@nhri.org.tw

${ }^{1}$ National Institute of Cancer Research, National Health Research Institutes, No. 367, Shengli Road, Tainan 704, Taiwan

${ }^{8}$ Graduate Institute of Medicine, College of Medicine, Kaohsiung Medical University, Kaohsiung 807, Taiwan

Full list of author information is available at the end of the article
}

transduction [1, 2]. PRMTs introduce monomethylation as well as symmetric or asymmetric dimethylation on their substrates by using $S$-adenosyl-L-methionine (SAM) as the methyl donor. Among the nine identified PRMTs in mammalian cells, PRMT3 is unique in several ways. First, PRMT3 contains a $\mathrm{C} 2 \mathrm{H} 2$ zinc finger domain that is not presented in other PRMTs and this domain is crucial for substrate recognition [3]. Second, PRMT3 is localized predominantly (or exclusively) in the cytoplasm under physiological circumstances, while other PRMTs are distributed both in the nucleus and cytoplasm or

(C) The Author(s). 2019 Open Access This article is distributed under the terms of the Creative Commons Attribution 4.0 International License (http://creativecommons.org/licenses/by/4.0/), which permits unrestricted use, distribution, and 
shuttled between these two compartments [3-5]. Although PRMT8 has also been suggested to be a cytosolic protein and may be recruited to the plasma membrane via myristoylation-mediated attachment, subsequent studies demonstrated that it is predominantly found in the nuclei of neuronal cells [6, 7]. Third, no histone proteins have been found to be methylated by PRMT3 in vivo until now. The existence of PRMTs in the nucleus suggests the possibility that these enzymes may directly methylate histone proteins to regulate gene expression via epigenetic modification. For instance, the methylation of histone $\mathrm{H} 4$ at arginine 3 (H4R3) is frequently detected in eukaryotic cells and this methylation is mainly catalyzed by PRMT1 [8]. Another histone marker H3R17 has been shown to be methylated by PRMT4, and the methylation plays a critical role in the induction of class II major histocompatibility genes by interferon- $\gamma$ [9]. A recent study demonstrated that PRMT6 methylates H3R2 to induce a global DNA hypomethylation by attenuating the recruitment of DNA methyltransferase 1 accessary factor UHRF1 to histone $\mathrm{H} 3$ [10]. To date, no arginine residues of histone proteins have been shown to be specifically methylated by PRMT3 in vivo.

The biological function of PRMT3 remains elusive due to the limited physiological substrates identified. Two previous studies demonstrated that the 40S ribosomal protein S2 (rpS2) is an in vivo PRMT3 substrate [11, 12]. The results showed that PRMT3 interacted with rpS2 via the zinc finger domain and methylated rpS2 in vitro. Interestingly, the 40S:60S free ribosomal subunit ratio was changed while the processing of pre-ribosomal RNA was largely unaffected in PRMT3-depleted cells. The knockout of PRMT3 in mice did not influence viability, although the animal size was smaller [13]. The methylation of rpS2 in PRMT3-deficient mice is indeed dramatically reduced suggesting that rpS2 is a physiological substrate of PRMT3. Additional reported PRMT3 substrates include Src-associated substrate during mitosis 68Kd (Sam68), poly(A)-binding protein 1 (PABP1), PABP2, nuclear poly(A)-binding protein (PABPN1), highmobility group A1, and p53 [14-18]. However, methylation of these proteins by PRMT3 was mainly demonstrated in vitro and the biological consequences induced by methylation in vivo were largely uncharacterized. By using gain-offunction mutant PRMT3 and modified SAM analogs as tools, a recent study identified 83 potential PRMT3 substrates in HEK293T cells [19]. Those substrates are known to be involved in the regulation of various cellular pathways, and four proteins including tubulin alpha- $1 \mathrm{C}$ chain (TUBA1C), TUBB4A, triosephosphate isomerase (TPI), and keratin type II cytoskeletal 6A (KRT6A) were further validated as PRMT3 substrates by biochemical approaches. However, the role of these substrates in PRMT3-mediated biological effects remains unclear.
In this study, we show that PRMT3 is upregulated in pancreatic cancer and is associated with poor patient survival, suggesting a novel oncogenic function of PRMT3. Moreover, we identified a total of 293 PRMT3interacting proteins in pancreatic cancer cells and found that PRMT3 methylated GAPDH at arginine 248 to promote glycolysis and mitochondrial respiration simultaneously in cancer cells. The combination of inhibitors of GAPDH and oxidative phosphorylation significantly suppresses cell proliferation in vitro and tumor growth in vivo.

\section{Materials and methods}

\section{Antibodies, chemicals, and plasmids}

Antibodies used were as follows: $\alpha$-GFP (Abcam \#ab290, Cambridge, UK), $\alpha$-GFP Sepharose (Abcam \#ab69314), $\alpha$-PRMT3 (GeneTex \#GTX23765, Irvine, CA, USA), $\alpha$ asymmetrical dimethyl arginine ( $\alpha$-ADMA) (Cell Signaling Technology \#13522, Denvor, MA, USA), $\alpha$-GAPDH (GeneTex \#GTX100118), $\alpha$-Flag (Sigma, \#F1804, St Louis, MO, USA), and $\alpha$-Actin (Millipore \#MAB1501, Birlington, MA, USA). Chemicals were as follows: SGC707 (Cayman \#17017, Ann Arbor, MI, USA), cycloheximide (Sigma \#C7698), heptelidic acid (BioVision \#2215-250, Milpitas, CA, USA), and oligomycin A (Cayman \#11342). Plasmids were as follows: The pEGFPPRMT3 expression vector was kindly provided by Dr. Mien-Chie Hung [20]. pcDNA3-PRMT3 expression vector was a gift from Dr. Jian Jin. Human GAPDH cDNA ORF Clone was purchased from Sino Biological (\#HG10094-NF, Beijing, China). R248K-GAPDH mutant was generated using a QuickChange site-directed mutagenesis kit according to the manufacturer's protocol (Agilent Technologies \#200519, Santa Clara, CA, USA). The primers used for mutagenesis are shown as follows $\left(5^{\prime}-3^{\prime}\right)$ :

\section{F: GTGGTGGACCTGACCTGCAAGCTAGAAAAAC CTGCC \\ R: GGCAGGTTTTTCTAGCTTGCAGGTCAGGTCC ACCAC}

\section{Cell culture and stable cell lines}

PANC-1 and HEK293T cells were cultured in DMEM medium with $10 \%$ fetal bovine serum (FBS) and $1 \%$ penicillin/streptomycin. PANC-1 cells with stable expressions of GFP and GFP-PRMT3 were generated in our lab and maintained in the DMEM medium supplemented with $800 \mu \mathrm{g} / \mathrm{ml} \mathrm{G418.} \mathrm{GFP/wild-type} \mathrm{GAPDH,}$ GFP-PRMT3/wild-type GAPDH, or GFP-PRMT3/ R248K-GAPDH mutant co-expressing PANC-1 stable cells was established in our lab and maintained in DMEM medium containing $800 \mu \mathrm{g} / \mathrm{ml}$ of $\mathrm{G} 418$ and $200 \mu \mathrm{g} / \mathrm{ml}$ hygromycin B. HPDE cells were kindly provided by Dr. Wun-Shaing Wayne Chang (National 
Institute of Cancer Research, National Health Research Institutes). HPDE cells were grown in keratinocyte serum-free media (Invitrogen, \#17005-042, Carlsbad, CA, USA) supplemented with bovine pituitary extract $(25 \mathrm{mg})$, EGF $(2.5 \mu \mathrm{g})$, and $1 \%$ penicillin/streptomycin. BxPC3 cells were kindly provided by Dr. Kuang-Hung Cheng [21]. BxPC3 cells were cultured in RPMI 1640 medium containing $2 \mathrm{mM}$ glutamine, 10\% FBS, and $1 \%$ penicillin/streptomycin. Miapaca-2 cells were grown in in DMEM medium with $10 \% \mathrm{FBS}, 2.5 \%$ horse serum, and $1 \%$ penicillin/streptomycin. Capan-2 cells were a gift from Dr. Wun-Shaing Wayne Chang and maintained in McCoy's 5a medium supplemented with 10\% FBS and $1 \%$ penicillin/streptomycin. L3.6pl cells were kindly provided by Dr. Mien-Chie Hung [22]. L3.6pl cells were cultured in DMEM/F12 medium containing 10\% FBS and $1 \%$ penicillin/streptomycin. Cell line identities were verified by short tandem repeat analysis and were confirmed as Mycoplasma free.

\section{Patient tumor tissue samples and immunoblotting}

Human pancreatic tumor tissues were obtained from patients undergoing surgical resection at Koo Foundation Sun Yat-Sen Cancer Center (Taipei, Taiwan) and National Cheng Kung University Hospital (Tainan, Taiwan) under the guidelines approved by the Institution Review Board at National Health Research Institutes. Written informed consent was obtained from each patient. Total proteins were extracted from human pancreatic tumor tissues using AllPrep DNA/RNA/Protein mini kits (Qiagen \#80004, Hilden, Germany) following the manufacturer's instructions. Briefly, tissues were lysed and homogenized in buffer RLT by using TissueRuptor. The lysates were centrifuged at 13,000 rpm for $3 \mathrm{~min}$, and the supernatant was passed through an AllPrep DNA spin column, which allows the binding of genomic DNA. Ethanol was added to the flow-through from the AllPrep DNA spin column, and the mixture was subsequently passed through an RNeasy spin column to collect total RNA. The supplied aqueous protein precipitation solution, buffer APP, was added into the flow-through of RNeasy spin column and incubated at room temperature for $10 \mathrm{~min}$, followed by centrifugation at 13,000 rpm for $10 \mathrm{~min}$. The precipitated protein pellets were resuspended by $500 \mu \mathrm{l}$ of $70 \%$ ethanol and were centrifuged at $13,000 \mathrm{rpm}$ for $1 \mathrm{~min}$. The total proteins were resuspended in 50-100 $\mu$ l buffer ALO, and equal amounts of proteins were subjected to western blot as described previously [23].

\section{Immunohistochemical (IHC) staining}

Human PDAC tissues were obtained from patients with surgical resection in National Cheng Kung University Hospital (Tainan, Taiwan) under the guidelines approved by the Institutional Review Board of National Cheng Kung University Hospital. Tissue sections were stained with PRMT3 (GeneTex \#GTX23765) antibody overnight at $4{ }^{\circ} \mathrm{C}$ followed by incubation with horseradish peroxidase (HRP)-conjugated secondary antibodies for $1 \mathrm{~h}$ at room temperature. The protein signal was developed using a 3,3'-diaminobenzidine solution.

\section{Mass spectrometry analysis}

GFP-PRMT3 proteins were purified from GFP-PRMT3overexpressing PANC-1 cells by immunoprecipitation with GFP antibody. The immunoprecipitated complexes were subjected to in-solution digestion with trypsin, and the PRMT3-interacting proteins were identified by mass spectrometry (Mithra Biotechnology Inc., Taiwan). To identify the arginine residue on GAPDH methylated by PRMT3, endogenous GAPDH proteins were purified from GFP-PRMT3-overexpressing PANC-1 cells by immunoprecipitation with GAPDH antibody and the immunoprecipitated complexes were separated by SDSPAGE. The protein bands corresponding to GAPDH were excised and subjected to in-gel digestion with trypsin. The samples were reduced in $50 \mathrm{mM}$ dithiothreitol at $37^{\circ} \mathrm{C}$ for $1 \mathrm{~h}$. Alkylation was conducted using $100 \mathrm{mM}$ iodoacetamide for $30 \mathrm{~min}$ in dark at room temperature. The resulting proteins were digested with trypsin at $37^{\circ} \mathrm{C}$ overnight. After digestion, the protein fragments were extracted with $10 \%$ formic acid and analyzed by liquid chromatography/tandem mass spectrometry (Mithra Biotechnology Inc., Taiwan).

\section{Metabolite extraction and metabolome analysis}

The cells were washed twice by using $5 \%$ mannitol solution and were then incubated with $800 \mu \mathrm{l}$ of methanol at room temperature to inactivate enzymes. The cell extracts were mixed with $550 \mu \mathrm{l}$ of Milli-Q water containing internal standard solution (Human Metabolome Technologies (HMT), H3304-1002) and incubated at room temperature for $30 \mathrm{~s}$. The extracted solutions were transferred into microtubes and centrifuged at $2300 \times g$, $4^{\circ} \mathrm{C}$ for $5 \mathrm{~min}$. The supernatant $(800 \mu \mathrm{l})$ was transferred to Millipore 5-kDa cutoff filter (UltrafreeMC-PLHCC, HMT), and the filters were centrifuged at $9100 \times g, 4{ }^{\circ} \mathrm{C}$ for $2-5 \mathrm{~h}$ until no liquid remained in the filter cup. The extracted sample solutions were completely evaporated and resuspended in $50 \mu \mathrm{l}$ of Milli-Q water for metabolome analysis at HMT. Metabolome analysis was performed by Basic Scan package of HMT using capillary electrophoresis time-of-flight mass spectrometry $(\mathrm{Hu}-$ man Metabolome Technologies, Inc., Tokyo, Japan)

\section{GAPDH activity assay}

The GAPDH activity was assayed in whole cells using a commercial GAPDH activity assay kit (BioVision \#680- 
100, Milpitas, CA, USA). Briefly, $5 \times 10^{5}$ cells were homogenized with $100 \mu \mathrm{l}$ of GAPDH assay buffer. Samples were kept on ice for $10 \mathrm{~min}$ and centrifuged at 10,000 $\times g$, $4^{\circ} \mathrm{C}$ for $5 \mathrm{~min}$. The GAPDH activity in the supernatants was studied according to the manufacturer's protocol. The absorbance at $450 \mathrm{~nm}$ was measured every $10 \mathrm{~min}$ for $1 \mathrm{~h}$. The experiments were done in triplicates and were repeated three times.

\section{ECAR and OCR measurement}

Extracellular acidification rate (ECAR) and oxygen consumption rate (OCR) were measured by extracellular flux (XF24) analyzer (Seahorse Bioscience) using glycolysis stress test kit (Agilent Technologies \#103020-100) and cell mito stress test kit (Agilent Technologies $\# 103015-100)$, respectively. Briefly, cells were seeded at $2 \times 10^{4}$ cells per well in XF24 plates in $100 \mu$ l of culture medium and incubated for $16-20 \mathrm{~h}$ at $37^{\circ} \mathrm{C}$ and $5 \% \mathrm{CO}_{2}$ prior to assay. For ECAR measurement, cell medium was replaced by XF assay medium supplemented with 2 $\mathrm{mM}$ glutamine and incubated at the incubator without supplied $\mathrm{CO}_{2}$ for $1 \mathrm{~h}$ before the completion of probe cartridge calibration. Basal ECAR was measured in the $\mathrm{XF}$ assay medium without glucose, and glycolysis was measured by injecting glucose $(10 \mathrm{mM})$, oligomycin $(1 \mu \mathrm{M})$, and 2-deoxy glucose $(50 \mathrm{mM})$ from XF24 reagent ports as indicated. For OCR measurement, cell medium was replaced by the $2 \%$ FBS culture medium and incubated at the incubator without $\mathrm{CO}_{2}$ for $1 \mathrm{~h}$ before the completion of probe cartridge calibration. Basal oxygen consumption rate (OCAR) was measured after injection of oligomycin $(1 \mu \mathrm{M})$, carbonyl cyanide-4-(trifluoromethoxy) phenylhydrazone $(0.5 \mu \mathrm{M})$, and rotenone $(2 \mu \mathrm{M})$.

\section{Drug synergy analysis}

For drug combination experiments, cells were treated with heptelidic acid or oligomycin for $48 \mathrm{~h}$ to determine the concentration that induced a 50\% inhibition of cellular growth (IC50) in the MTT assay. Heptelidic acid was combined with oligomycin at a constant ratio determined by $\mathrm{IC}_{50}$ Heptelidic $A$ cid $/ \mathrm{IC}_{50}$ Oligomycin. Inhibition of cell growth by the combination of these two inhibitors was measured by MTT assay. The effects of drug combinations were evaluated with Calcusyn software (Biosoft) according to Chou-Talalay combination index method [24]. $\mathrm{CI}>1$ indicates antagonism, $\mathrm{CI}=1$ indicates additive effect, and $\mathrm{CI}<1$ indicates synergism. All experiments were carried out in triplicate.

\section{Xenograft animal experiments}

All animal experiments were approved by Animal Care Committee of National Health Research Institutes. Advanced severe immunodeficiency (ASID) mice at 4-5 weeks were housed under standard conditions. GFPand GFP-PRMT3-overexpressing PANC- 1 cells $\left(1 \times 10^{6}\right)$ were suspended in $50 \mu \mathrm{l}$ PBS mixed with $50 \mu$ l Matrige and subcutaneously injected into the right flank of the mice. Tumor burden was monitored with digital calipers twice per week, and tumor volume was estimated using the formula (length $\times$ width $\left.^{2}\right) / 2$. Three weeks after injection, mice were randomly divided into two groups to receive PBS (control) and oligomycin $(0.5 \mathrm{mg} / \mathrm{kg})+$ heptelidic acid $(1 \mathrm{mg} / \mathrm{kg})$. The number of mice per group was five. All of the mice received the drugs via tumor injection twice per week. After 1 week, tumors were harvested and tumor weight was measured.

\section{TUNEL assay}

Apoptosis of tumor tissues was analyzed using terminal deoxynucleotidyl transferase-mediated dUTP nick end labeling (TUNEL) assay (Abcam \#ab66110) according to the manufacturer's instruction. Sections were analyzed using a Leica DMi8 microscope (Leica Microsystems, Inc.). The percentage of cell death was determined by counting the number of TUNEL-positive cells in three independent fields of different slides using ImageJ software.

\section{Quantification and statistical analysis}

Results were shown as the Means $\pm \operatorname{SEM}(n=3)$. Differences between various experimental groups were evaluated by using a two-tailed, unpaired Student's $t$ test, and $p$ value less than 0.05 was considered as statistically significant.

\section{Results}

PRMT3 is overexpressed in pancreatic cancer and is associated with poor clinical outcome

To verify the clinical significance of PRMT3, we compared the expression of PRMT3 in immortalized human pancreatic ductal epithelial (HPDE) cells and human pancreatic cancer cell lines and found that PRMT3 was upregulated in most of cancer cell lines (Fig. 1a). In addition, the increase of PRMT3 was detected in 69\% $(11 / 16)$ of the pancreatic tumor tissues investigated (Fig. 1b). Semi-quantification of the protein level by densitometry demonstrated that tumor tissues have $>2$ fold increase of PRMT3 when compared to the averaged level of four adjacent normal tissues (Fig. 1b). Immunohistochemical staining showed that PRMT3 protein is mainly detected in ductal cells and its expression is significantly increased in tumor tissues (Fig. 1c). Moreover, analysis of PRMT3 expression in the 176 pancreatic cancer patients published in The Cancer Genome Atlas (TCGA) database demonstrated that high PRMT3 expression is an unfavorable prognostic factor and is associated with reduced patient survival (Fig. 1d, data 


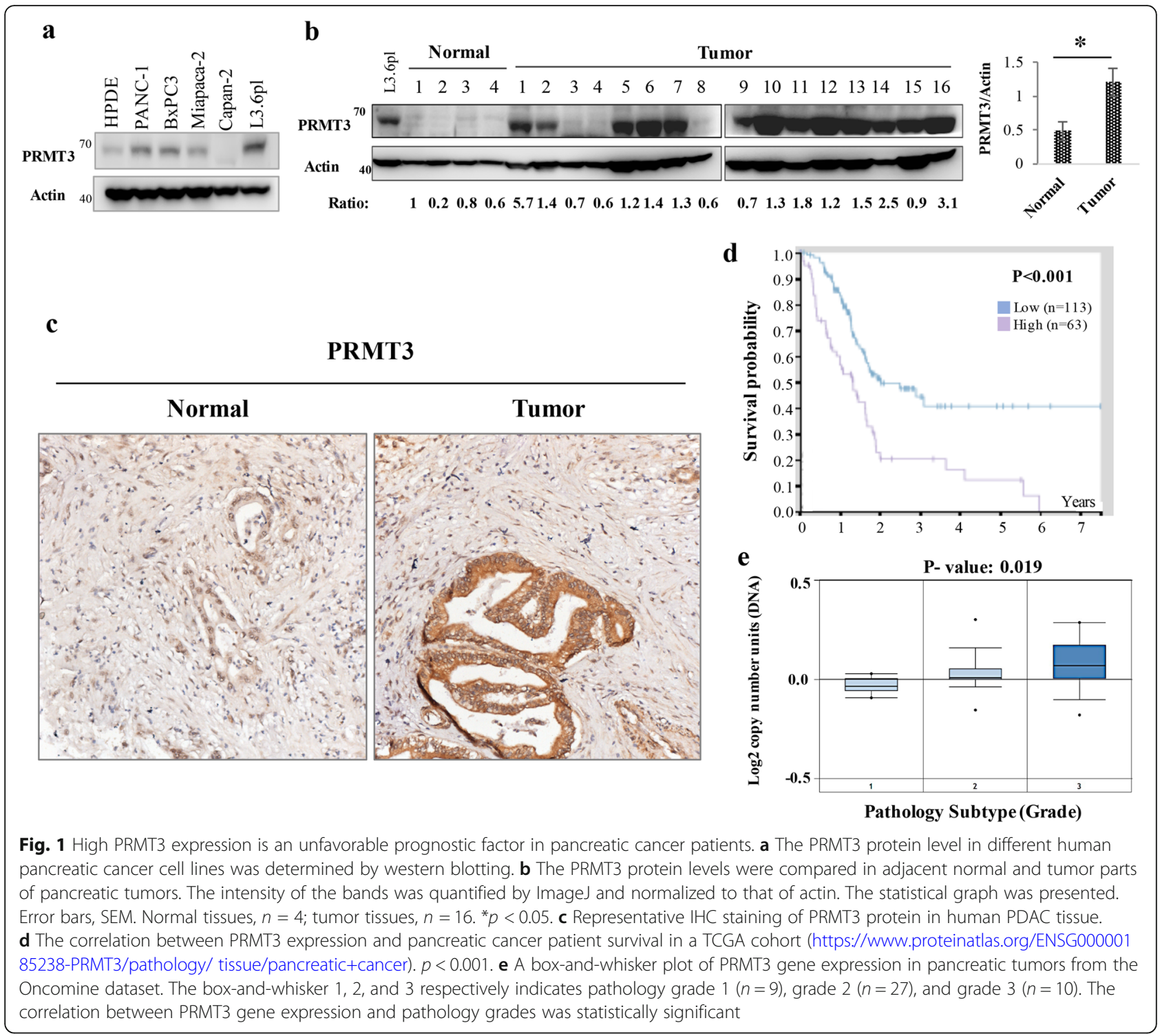

derived from https://www.proteinatlas.org/ENSG000001 85238-PRMT3/pathology/ tissue/pancreatic+cancer of The Human Protein Atlas) [25]. Additionally, increased PRMT3 expression is found in high-grade tumors in the Oncomine dataset (Fig. 1e). These data suggested an oncogenic role of PRMT3 in pancreatic cancer.

\section{GAPDH is an in vivo substrate of PRMT3}

To elucidate the biological function of PRMT3, we sought to identify its interacting proteins in pancreatic cancer cells. Green fluorescent protein (GFP)-tagged PRMT3 was ectopically expressed in PANC-1 cells, and the associated proteins were pulled down for proteomics analysis (Fig. 2a). A total of 293 proteins including rpS2, a confirmed substrate of PRMT3, were identified (Additional file 1: Table S1). In agreement with previous results [19], PRMT3 was found to be associated with a number of metabolic enzymes, consistent with its cytosolic location (Fig. 2b). Three interacting proteins including GAPDH, glucose-6-phosphate isomerase (G6PI), and citrate dehydrogenase (CISY) were identified in both HEK297T [19] and PANC-1 (this study) cells. We focused on GAPDH, and the interaction between PRMT3 and GAPDH was validated by immunoprecipitation/immunoblotting assay (Fig. 2c). More importantly, we detected the asymmetric dimethylarginine (ADMA) methylation of GAPDH in PANC-1 cells with ectopic expression of PRMT3 (Fig. 2d, left upper panel). The treatment of PRMT3 inhibitor SGC707 reduced the ADMA signal of GAPDH in L3.6pl cells (Fig. 2d, right upper panel). In addition, PRMT3 knockdown in L3.6pl cells decreased the ADMA signal of GAPDH (Fig. 2d, bottom lower panel). These results suggested that GAPDH could be a physiological substrate of PRMT3. 


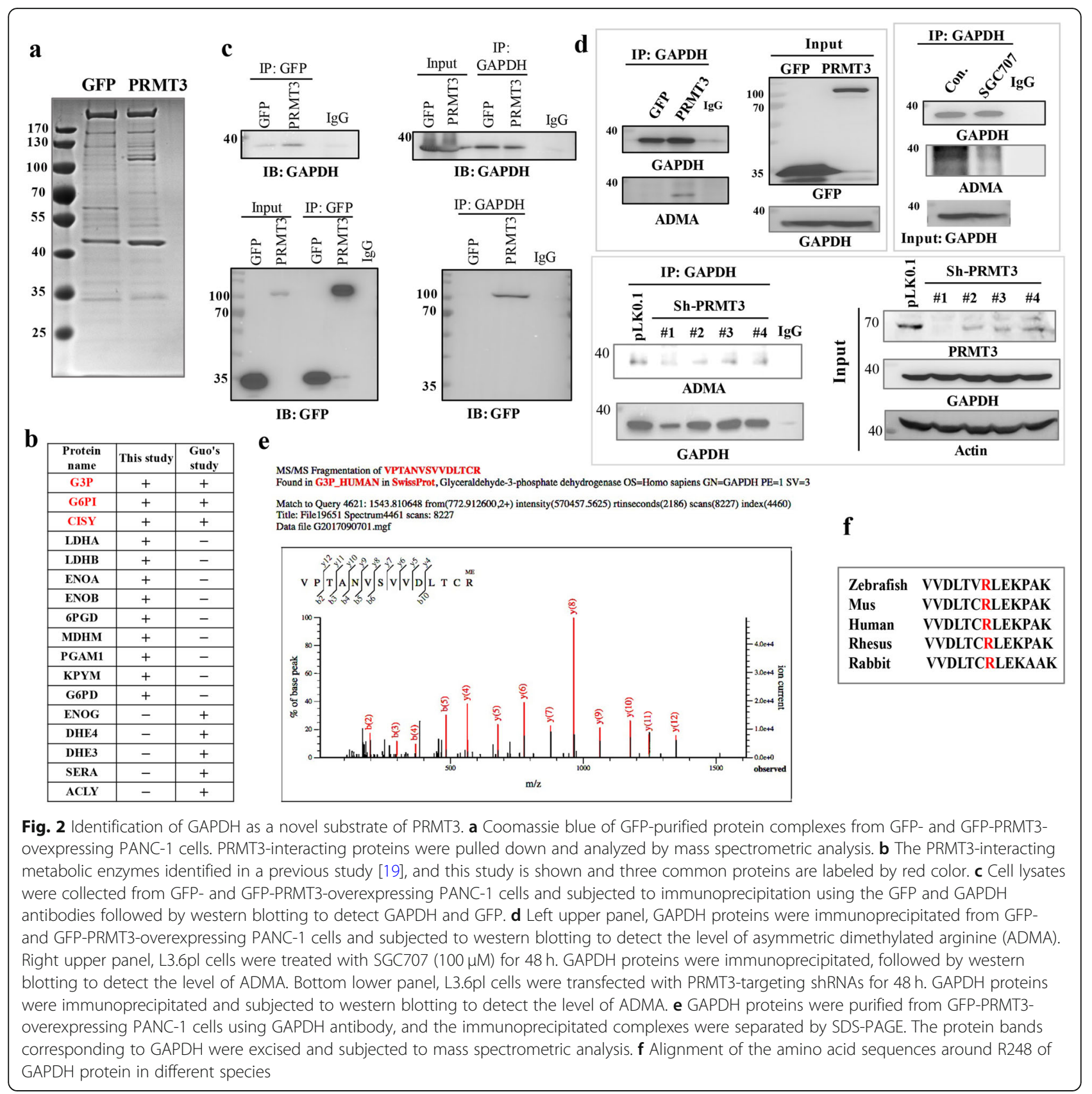

Liquid chromatography coupled with tandem mass spectrometry (LC-MS/MS) identified a single methylation site at Arg248 (R248) (Fig. 2e). Sequence alignment demonstrated that this arginine residue is highly conserved in different species, indicating the methylation of this residue may have important biological significance (Fig. 2f).

\section{Methylation of R248 enhances the catalytic activity of GAPDH}

We found the GAPDH activity was increased by threefold in the PRMT3-overexpressing PANC-1 cells, and this increase was suppressed by the specific PRMT3 inhibitor SGC707. Moreover, PRMT3 knockdown in L3.6pl cells decreased GAPDH activity, suggesting PRMT3-mediated methylation of GAPDH may upregulate its catalytic activity (Fig. 3a). Mutation of Arg (R) to Lys $(\mathrm{K})$ retains the positive charge of $\mathrm{R}$ and creates a residue that cannot be methylated by PRMT3 [26]. We generated the R248K mutant GAPDH and compared its catalytic activity with wild-type enzyme after expression in HEK293T cells. Our data showed that the activity of the R248 mutant was very low (Fig. 3b, left panel). Ectopic expression of the R248 mutant in human $\mathrm{L} 3.6 \mathrm{pl}$ 


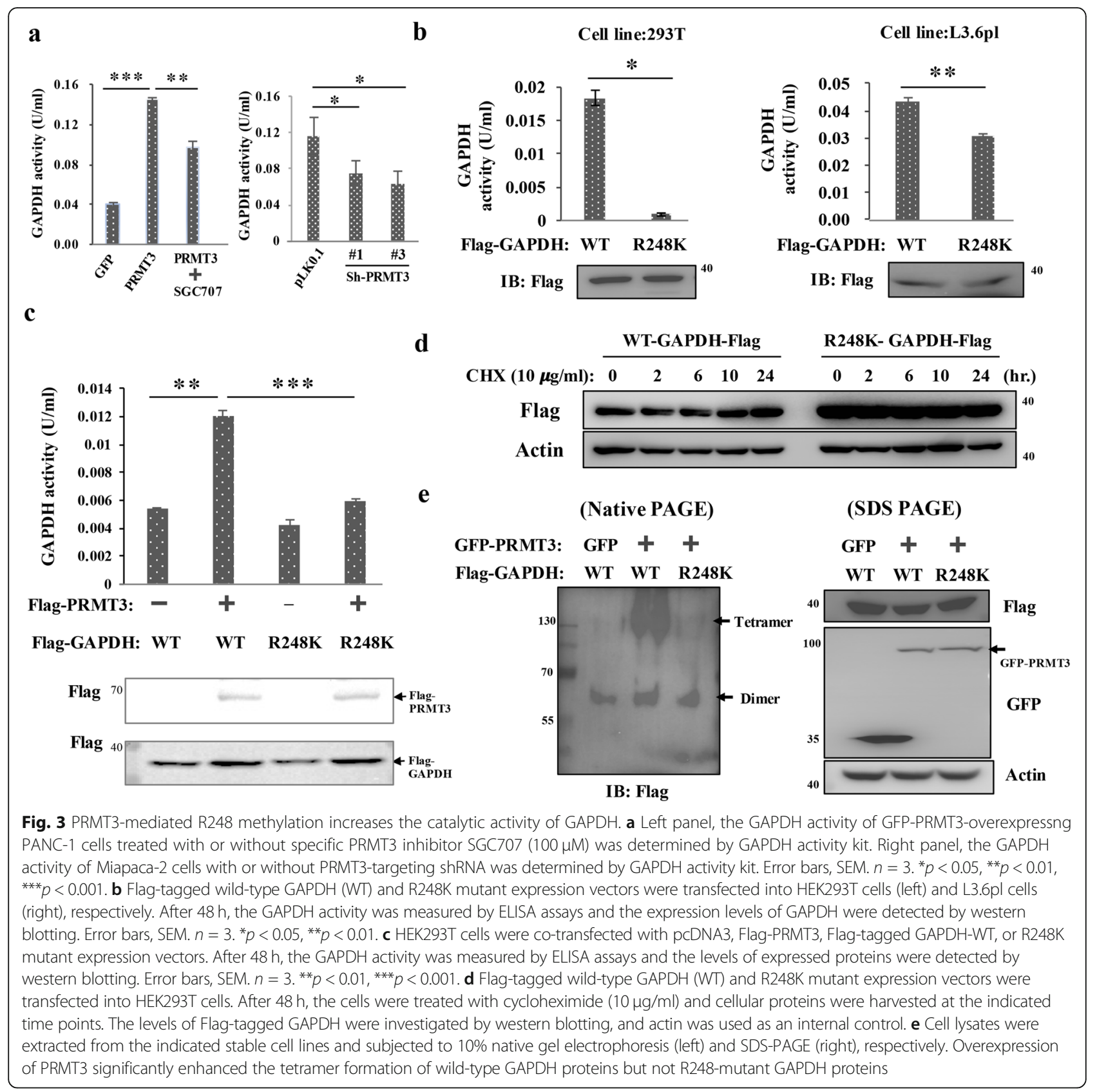

pancreatic cancer cells, which express abundant endogenous PRMT3, also decreased the total GAPDH activity in cells (Fig. 3b, right panel). Because active GAPDH is a homotetramer protein complex [27], our results suggested that the R248 mutant may interfere the assembly or activity of active tetramer. Mutation of R248 markedly reduced PRMT3-increased GAPDH activity in the HEK293T cells co-transfected with PRMT3 and GAPDH vectors (Fig. 3c). We next studied whether methylation of R248 changed the protein stability of GAPDH. Our data did not support the hypothesis because (1) the GAPDH protein levels in the control and PRMT3-overexpressing PANC-1 cells were similar (Fig. 2c) and (2) the stabilities of wild-type and R248K mutant GAPDH proteins in transfected HEK293T cells were also similar (Fig. 3d). We next tested the possibility that methylation of GAPDH at R248 by PRMT3 may promote the assembly of active tetramer. The result of native gel electrophoresis demonstrated that co-expression of PRMT3 and wild-type GAPDH increased the tetrameric form of GAPDH while co-expression of PRMT3 and GAPDH-R248K mutant did not (Fig. 3e). These data suggested that PRMT3-induced R248 methylation enhances GAPDH activity by promoting the assembly of active tetramer. 


\section{PRMT3-mediated methylation of GAPDH promotes metabolic reprogramming}

To address the biological consequence induced by PRMT3-mediated methylation of GAPDH, intracellular metabolites were analyzed by capillary electrophoresis time-of-flight mass spectrometry. We detected 174 metabolites in the control and PRMT3-overexpressing PNAC-1 cells, and principle component analysis revealed a significant difference of the metabolites in these two cell lines (Fig. 4a). Hierarchical cluster analysis also showed a dramatic alteration of intracellular metabolite levels (Fig. 4b). One of the most obviously altered pathways was the central carbon metabolism, with a significant increase of the intermediates in glycolysis and tricarboxylic acid cycle in PRMT3-overexpressing cells (Fig. 4c). In addition, the metabolism of lipids and amino acids was also upregulated, suggesting the activation of the pentose phosphate pathway (Fig. 4d). Two additional pathways affected were branched chain/aromatic amino acids and nucleotide metabolism, respectively (Additional files 2 and 3: Figures S1 and S2). Several coenzymes including NADH, NAPDH, and acetyl-coenzyme A were enriched in cells with PRMT3 overexpression (Additional file 4: Figure S3). Consistent with upregulation of glycolysis and mitochondrial respiration, the extracellular acidification rate (ECAR) and oxygen consumption rate (OCR) were both increased in PRMT3overexpressing PANC-1 cells and were significantly inhibited by SGC707 (Fig. 5a, b). In addition to PANC-1 cells, we also tested the effect of PRMT3 inhibition on the glycolysis and mitochondrial respiration in normal HPDE cells and L3.6pl and Capan-2 pancreatic cancer cells. The treatment of SGC707 inhibited ECAR and OCR levels of L3.6pl cells more significantly than that of HPDE and Capan-2 cells which express a low level of PRMT3 protein (Additional file 5: Figure S4). To confirm that PRMT3-mediated metabolic reprogramming is dependent on GAPDH methylation, we ectopically expressed the R248K mutant in PRMT3overexpressing PANC-1 cells and found that both ECAR and OCR were significantly suppressed (Fig. 5c, d). These data suggested that PRMT3 promotes glycolysis and mitochondrial respiration simultaneously via the methylation of GAPDH.

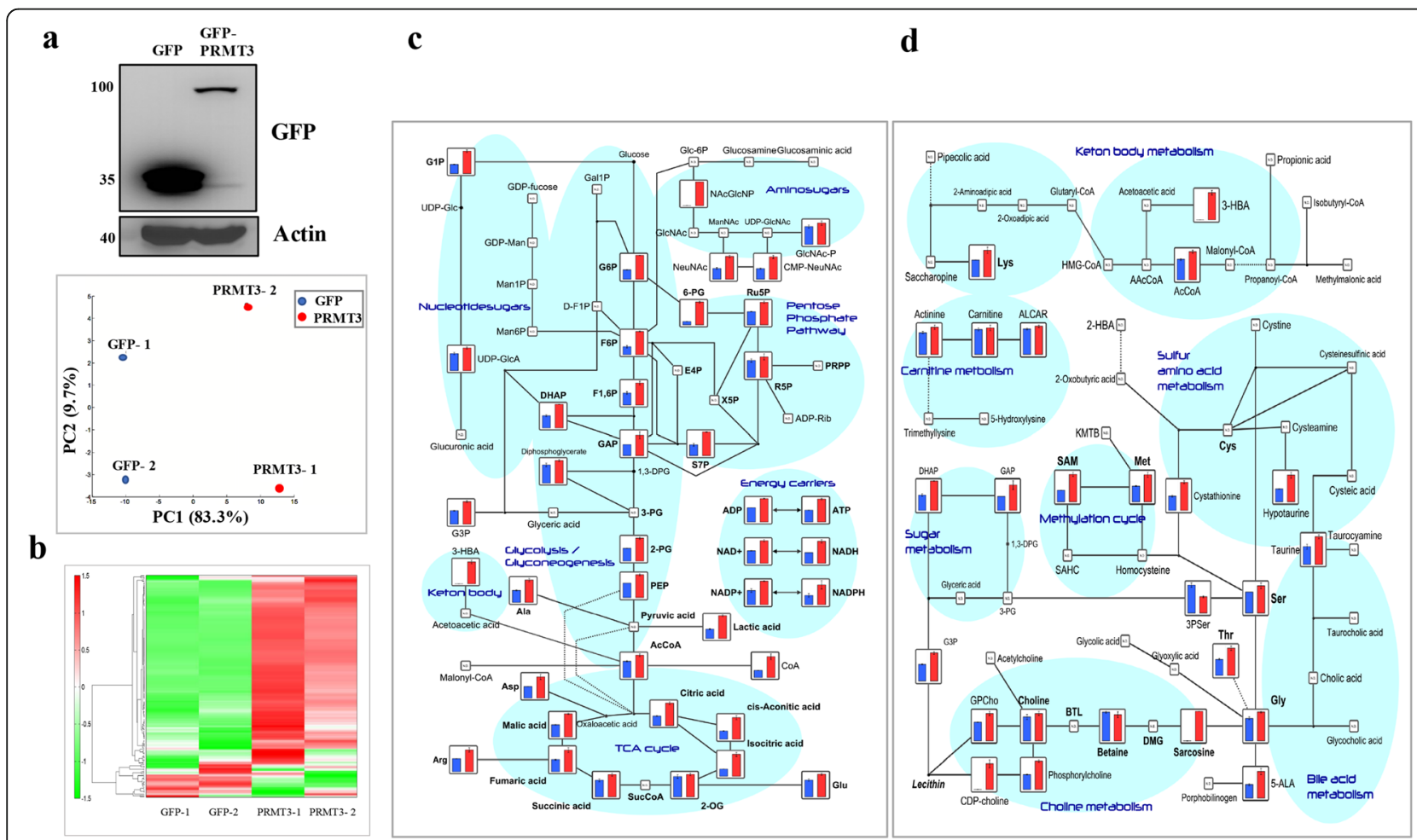

Fig. 4 Overexpression of PRMT3 induces metabolic reprogramming. a Principal component analysis of the metabolites in GFP- and GFP-PRMT3overexpressing PANC-1 cells. The expression levels of GFP and GFP-PRMT3 were detected by western blotting. b Hierarchical cluster analysis (HCA) of the metabolites in GFP- and GFP-PRMT3-overexpressing PANC-1 cells. The horizontal axis and vertical axis show sample names and peaks. c Change of the metabolites in central carbon metabolism. The bars/lines represent relative areas of each metabolite in GFP- (blue) and GFP-PRMT3 (red)-overexpressing PANC-1 cells, respectively. N.D., not detected. $\mathbf{d}$ Change of the metabolites in lipid and amino acid metabolism. The bars/lines represent relative areas of each metabolite in GFP- (blue) and GFP-PRMT3 (red)-overexpressing PANC-1 cells, respectively. N.D., not detected 


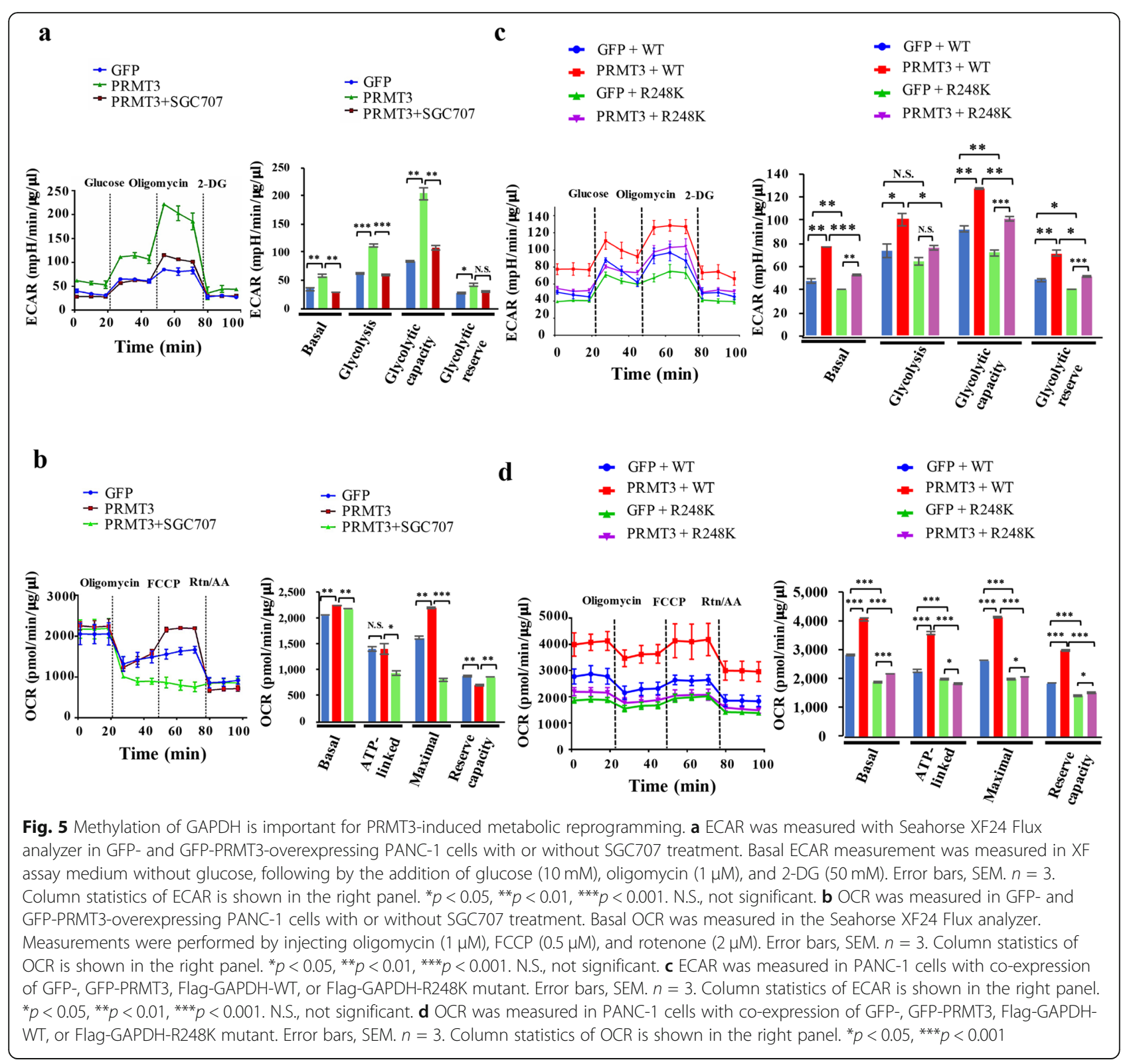

\section{Overexpression of PRMT3 sensitizes pancreatic cancer cells to GAPDH blockade}

Because GAPDH is an important effector for PRMT3 to reprogram cellular metabolism, we hypothesized that PRMT3-overexpressing pancreatic cancer cells may be addicted to GAPDH for proliferation. Indeed, PRMT3overexpressing PANC-1 cells were more sensitive to the GAPDH inhibitor heptelidic acid than the parental cells (Fig. 6a). Heptelidic acid also suppressed the proliferation of BxPC3 and PANC-1 pancreatic cells more significantly than that of normal HPDE cells (Fig. 6b). A unique feature of PRMT3-induced metabolic reprogramming is the simultaneous upregulation of glycolysis and mitochondrial respiration. Therefore, we tested whether the combination of oligomycin (an F0/F1 ATP synthase and mitochondrial respiration inhibitor) with heptelidic acid could elicit a more significant growth-suppressive effect. Combination index analysis confirmed these two inhibitors synergistically suppressed the proliferation of BxPC3 and L3.6pl pancreatic cancer cells (Fig. 6c). Finally, we validated the synergistic effect of oligomycin and heptelidic acid in vivo. GFP- and GFP-PRMT3overexpressing PANC-1 cells were subcutaneously injected into the mice, and the mice were treated without or with combined drugs after tumor formation. Although we did not find a significant increase of tumor growth in the animals injecting with PRMT3-overexpressing PANC-1 cells, the percentage of Ki-67positive cells in the tumors was increased (Additional file 6: Figure S5). Combination of oligomycin with 


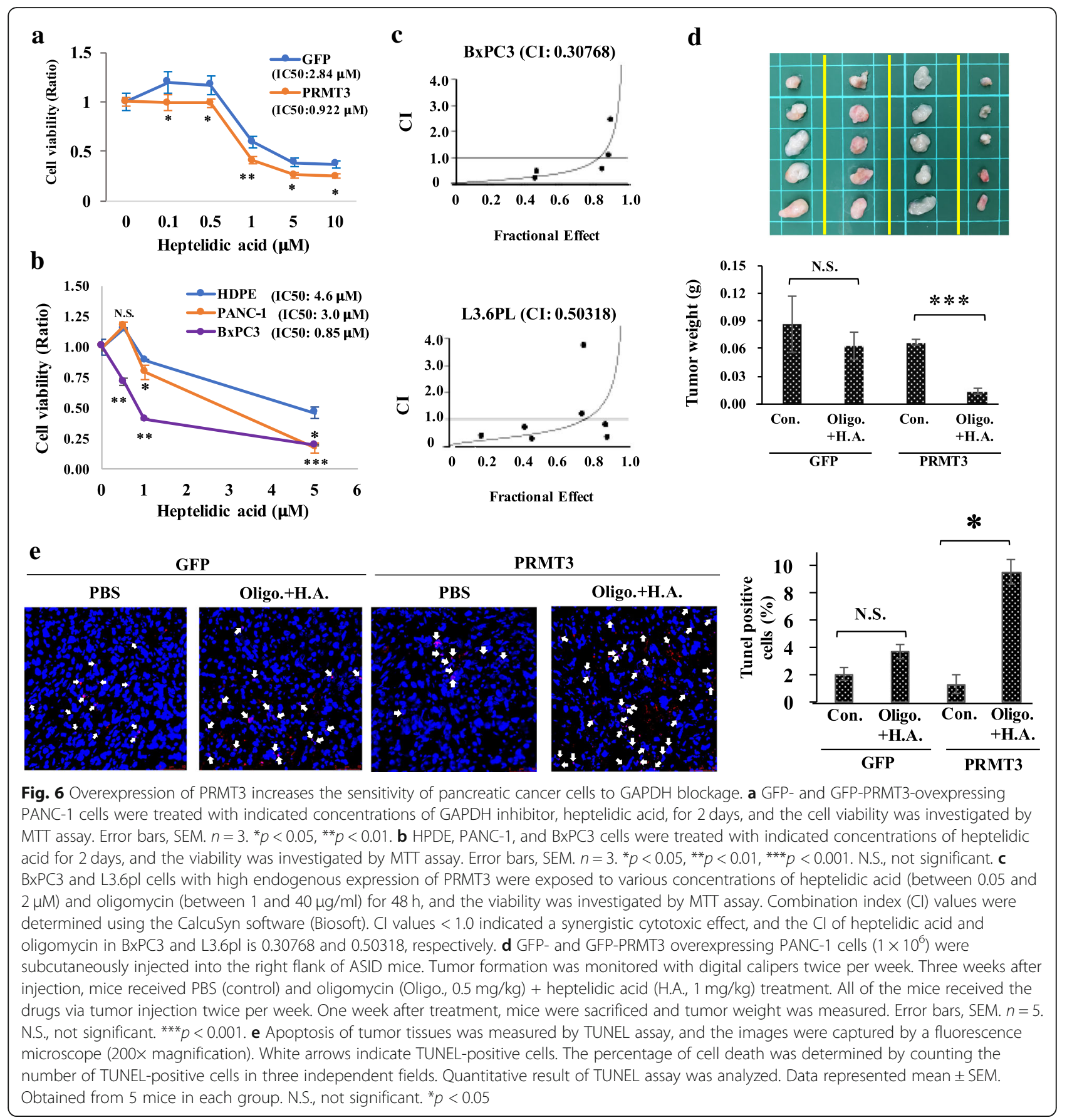

heptelidic acid significantly suppressed tumor growth of PRMT3-overexpressing cancer cells but not that of parental PANC-1 cells (Fig. 6d). In addition, drug combination only triggered a significant increase of apoptotic cells in the PRMT3-overexpressing tumors (Fig. 6e). In another animal study, depletion of PRMT3 in Miapaca-2 pancreatic cells decreased tumor growth in vivo and increased cancer cell apoptosis in the tumor tissues (Additional file 7: Figure S6). These data suggested that PRMT3overexpressing cancer cells are susceptive to double blockade of GAPDH and mitochondria respiration in vitro and in vivo (Fig. 7, proposed model).

\section{Discussion}

Currently, the only cellular process confirmed to be regulated by PRMT3 in animals and plants is ribosomemediated protein biogenesis, because the ribosomal protein rpS2 has been shown to be a methylation substrate of PRMT3 [11, 28]. Although PRMT3 has been shown to enhance hepatic lipogenesis, this effect is methylation- 


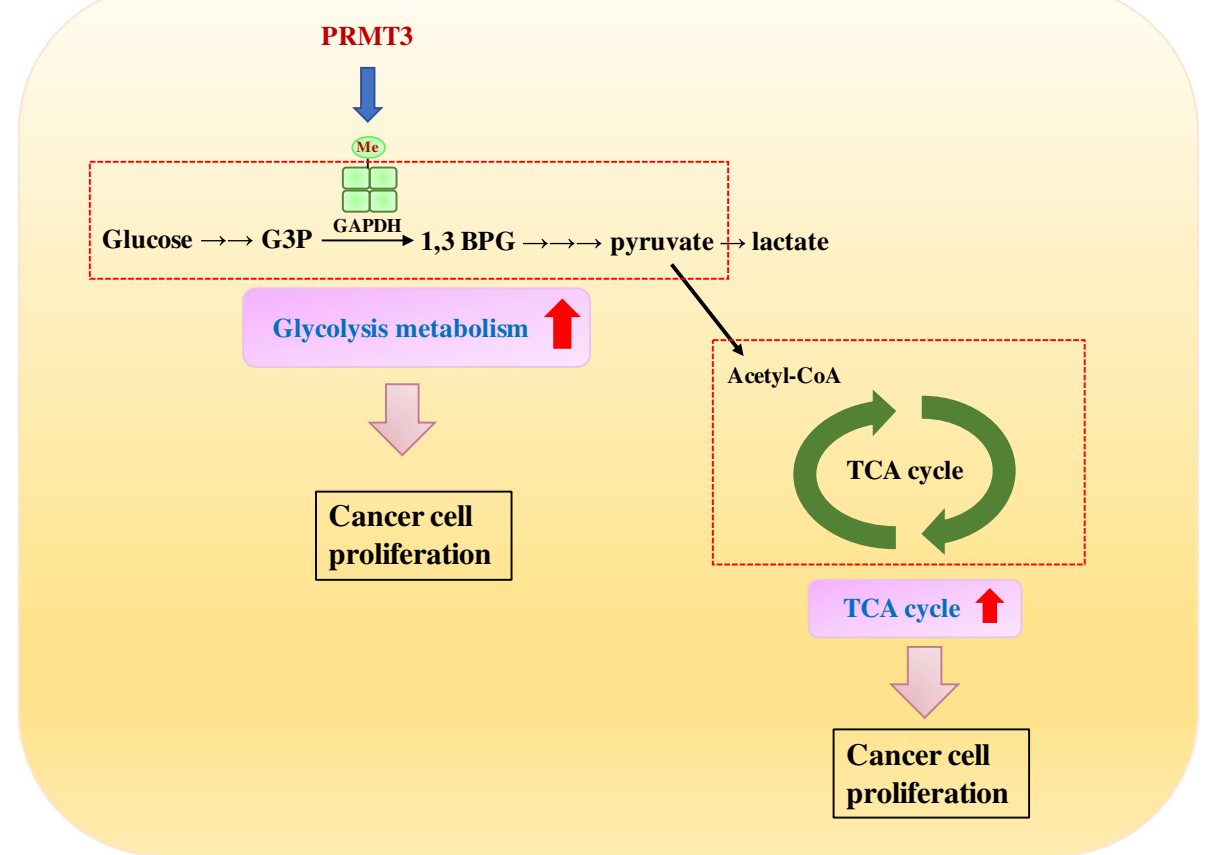

Fig. 7 Proposed model of PRMT3-mediated metabolic reprogramming in pancreatic cancer cells. PRMT3 methylates GAPDH at arginine 248 to promote glycolysis and mitochondrial respiration simultaneously in cancer cells. Double blockade of glycolysis and mitochondrial respiration could be a novel strategy for the treatment of PRMT3-overexpressing pancreatic cancer

independent and is mediated by direct interaction between PRMT3 and liver X receptor- $\alpha$, a nuclear receptor that controls the transcription of lipogenic enzymes like fatty acid synthase and acetyl-coenzyme A carboxylase [29]. In this study, we provide the first evidence that PRMT3 directly methylates GAPDH to promote glycolysis and mitochondrial respiration. The intermediates in the glycolytic pathway and tricarboxylic acid cycle are all increased in PRMT3-overexpressing cells. In addition, these cells exhibit increased ECAR and OCR, which can be reversed by ectopic expression of methylation-deficient R248K mutant GAPDH, confirming the importance of GAPDH in the regulation of cellular metabolism by PRMT3.

Posttranslational modifications (PTM) such as $S$-nitrosylation, acetylation, phosphorylation, and $\mathrm{O}$-linked $\mathrm{N}$ acetyl glucosamine modification of GAPDH have been demonstrated previously [30, 31]. However, little is known about arginine methylation of this glycolytic enzyme. When our study was undergoing, two studies reported that PRMT1 and PRMT4 could methylate GAPDH in cells $[32,33]$. Cho et al. demonstrated that PRMT1 induces arginine methylation of GAPDH, resulting in the inhibition of GAPDH $S$-nitrosylation and nuclear localization [32]. However, no methylation site was identified in the study. Zhong et al. showed that PRMT4 methylates GAPDH at R234 and suppresses its catalytic activity to suppress glycolysis and proliferation of liver cancer cells [33]. Our results indicate that R248 is the major residue methylated by PRMT3 in vivo, and R248 methylation enhances metabolic reprogramming and cellular proliferation of pancreatic cancer cells. R248 is located at the dimer interface, which plays a critical role in the formation of active tetramer [34]. It is possible that methylation at this residue may promote tetramer assembly or stabilize active tetramer. This hypothesis is supported by our finding that mutation of R248 significantly decreases tetramer formation (Fig. 3e) and dramatically reduces GAPDH activity (Fig. 3b, c). Another important issue to be considered is the synergy or antagonism between different PTMs adjacent to R248. The Cys247 (C247) residue of GAPDH has been shown to be modified by $S$-nitrosylation, and this PTM is stimulated by oxidized low-density lipoprotein and interferon- $\gamma$ [35]. Phosphorylation of Thr246 (T246) induced by protein kinase $\mathrm{C} \delta$ under the stress of cardiac ischemia and reperfusion increases the association of GAPDH with mitochondria and inhibits GAPDH-triggered mitophagy [36]. Functional interplay between phosphorylation and arginine methylation was firstly demonstrated in the transcription factor C/EBP $\beta$ [37]. Methylation of R3 in the $\mathrm{N}$-terminal transactivation domain of $\mathrm{C} / \mathrm{EBP} \beta$ by PRMT4 regulates the interaction of $C / E B P \beta$ with the SWI/SNF chromatin remodeling complex and alters the transcription of target genes. Interestingly, phosphorylation of T220 of C/EBP $\beta$ by mitogen-activated kinase 
attenuates PRMT4-mediated R3 methylation. These data suggest that phosphorylation may antagonize the effect of arginine methylation in the regulation of transcription factor activity. Whether the $S$-nitrosylation, phosphorylation, and arginine methylation at the 246-248 residues of GAPDH may occur independently, or simultaneously or consequently under various physiological or pathological circumstances, and whether the crosstalk between these PTMs may fine-tune GAPDH function to adapt extracellular alterations are important issues for further characterization.

Metabolic reprogramming is an important process for cancer cells to fit the high demand of energy requirement and supplementation of biosynthetic building blocks. Glycolysis is the metabolic pathway that converts one molecule of glucose to two molecules of pyruvate and generates two molecules of ATP and NADH per reaction. Although the efficiency of ATP production is low, the intermediates generated during the reactions could be used for synthesis of amino acids, lipids, and nucleotides to support rapid tumor growth. Therefore, many cancers switch their cellular metabolism to glycolysis under oxygen-rich conditions and the inhibition of the glycolytic pathway is considered to be a novel strategy for cancer therapy [38, 39]. However, recent studies point out that mitochondrial respiration also plays a critical role in the survival and metastasis of cancer cells [40]. In pancreatic cancer, inhibition of KRAS signaling induces extensive cancer cell death. However, a minor population of cancer cells with stemness properties may survive after oncogene ablation and those cells are highly dependent on mitochondrial respiration for survival and regrowth [41]. Similarly, chronic myeloid leukemia stem cells left after target therapy rely on mitochondrial metabolism for survival [42]. In addition, breast cancer cells may increase their invasive ability by upregulating peroxisome proliferator-activated receptor $\gamma$ coactivator $1 \alpha$-mediated mitochondrial biogenesis and oxidative phosphorylation [43]. An important finding of this study is the simultaneous increase of glycolysis and mitochondrial respiration in PRMT3-reprogrammed cells. This unique feature provides a molecular basis for the double blockade of these two metabolic pathways in attempts to kill PRMT3-overexpressing cancer cells. Indeed, the combination of oligomycin with heptelidic acid induces a synergistic antitumor effect in vitro and in vivo.

\section{Conclusion}

In this study, we show that PRMT3-mediated R248 methylation of GAPDH is critical for metabolic reprogramming and cellular proliferation, and double blockade of glycolysis and mitochondrial respiration could be a novel strategy for the treatment of PRMT3-overexpressing pancreatic cancer.

\section{Additional files}

Additional file 1: Table S1. PRMT3-associated proteins identified from mass spectrometry. (DOCX $36 \mathrm{~kb}$ )

Additional file 2: Figure S1. Change of the metabolites in branched chain and aromatic amino acids metabolism. The bars/lines represent relative areas of each metabolite in GFP- (blue) and GFP-PRMT3 (red)overexpressing PANC-1 cells, respectively. N.D., not detected. (PDF 229 kb)

Additional file 3: Figure $\mathbf{5 2}$. Change of the metabolites in nucleotide metabolism. The bars/lines represent relative areas of each metabolite in GFP- (blue) and GFP-PRMT3 (red)-overexpressing PANC-1 cells, respectively. N.D., not detected. (PDF $184 \mathrm{~kb}$ )

Additional file 4: Figure S3. Change of the metabolites in metabolism of coenzymes. The bars/lines represent relative areas of each metabolite in GFP- (blue) and GFP-PRMT3 (red)-overexpressing PANC-1 cells, respectively. N.D., not detected. (PDF 492 kb)

Additional file 5: Figure S4. The inhibition of PRMT3 suppresses ECAR and OCR levels. $(\mathrm{a}-\mathrm{C}) \mathrm{L}$ 3.6pl, HPDE, and Capan-2 cells were treated with SGC707 $(100 \mu \mathrm{M})$ for $48 \mathrm{~h}$. The ECAR and OCR levels were measured with Seahorse XF24 Flux analyzer. Error bars, SEM. $n=3$. (PDF 3078 kb)

Additional file 6: Figure S5. The combination of oligomycin and heptelidic acid significantly suppresses the growth of PRMT3-overexpressing cancer cells. Cell proliferation of tumor tissues was measured by Ki67 staining, and the images were captured by a microscope. The percentage of Ki67 staining was determined by counting the number of Ki67 positive cells in three independent fields. Quantitative result of Ki67 staining was analyzed. Data represented mean \pm SEM. Obtained from 5 mice in each group. ${ }^{*} p<0.05,{ }^{* * *} p<0.001$. (PDF $2377 \mathrm{~kb}$ )

Additional file 7: Figure S6. Advanced severe immunodeficiency (ASID) mice were housed under standard conditions. pLK0.1- and sh-PRMT3overexpressing Miapaca- 2 cells $\left(1 \times 10^{7}\right)$ were suspended in $50 \mu \mathrm{l}$ PBS mixed with $30 \mu$ l Matrige and subcutaneously injected into the left flank of the mice. Tumor burden was monitored with digital calipers twice per week. Two weeks after injection, tumors were harvested and tumor weight was measured. Apoptosis of tumor tissues was analyzed using terminal deoxynucleotidyl transferase-mediated dUTP nick end labeling (TUNEL) assay. The percentage of cell death was determined by counting the number of TUNEL-positive cells in three independent fields of different slides using ImageJ software. The results showed that PRMT3 knockout suppressed tumor growth and increased cell apoptosis. Data represented mean \pm SEM. Obtained from 5 mice in each group. (PDF 1917 kb)

\section{Abbreviations}

ADMA: Asymmetric dimethylarginine; Cl: Combination index;

ECAR: Extracellular acidification rate; GAPDH: Glyceraldehyde-3-phosphate dehydrogenase; HPDE: Human pancreatic ductal epithelial cells; OCR: Oxygen consumption rate; PRMTs: Protein arginine methyltransferases; SAM: SAdenosyl-L-methionine; TUNEL: Terminal deoxynucleotidyl transferasemediated dUTP nick end labeling

\section{Acknowledgements}

We thank Dr. Mien-Chie Hung, Dr. Jian Jin, and Dr. Wun-Shaing Wayne Chang for providing the experimental materials.

\section{Authors' contributions}

$\mathrm{MCH}$ and WCH designed the study. MCH, YLT, and $\mathrm{CHL}$ performed the experiments. MCH, YLT, MRP, and WCH analyzed and interpreted the experimental data. YSS, HCC, TYC, and LTC provided the discussion and suggestions to the experiments. MCH and WCH wrote the manuscript with input from all authors. All authors read and approved the final manuscript.

\section{Funding}

This work was supported by the grants from the Ministry of Science and Technology (MOST-103-2314-B-037-075, MOST-104-2314-B-037-004, MOST105-2314-B-037-001, and MOST-107-2320-B-400-006-MY3 to MRP and WCH and MOST-106-2321-B-400-007 to LTC). This study was also supported by the grant from the National Research Institutes (NHRI-107A1-CACO-02181811 to YSS) and the Yen-Hsu Education Foundation to WCH. 


\section{Availability of data and materials}

All data generated or analyzed during this study are included in this article and its additional files.

\section{Ethics approval and consent to participate}

Human pancreatic tumor tissues were obtained from patients undergoing surgical resection at Koo Foundation Sun Yat-Sen Cancer Center (Taipei, Taiwan) and National Cheng Kung University Hospital (Tainan, Taiwan) under the guidelines approved by the Institution Review Board at National Health Research Institutes. Written informed consent was obtained from each patient. All animal experiments were approved by Animal Care Committee of National Health Research Institutes.

\section{Consent for publication}

Not applicable.

\section{Competing interests}

The authors declare that they have no competing interests.

\section{Author details}

${ }^{1}$ National Institute of Cancer Research, National Health Research Institutes, No. 367, Shengli Road, Tainan 704, Taiwan. ${ }^{2}$ Institute of Clinical Medicine, College of Medicine, Kaohsiung Medical University, Kaohsiung 807, Taiwan. ${ }^{3}$ Institute of Clinical Medicine, National Cheng Kung University, Tainan 704, Taiwan. ${ }^{4}$ Department of Surgery, National Cheng Kung University Hospital, Tainan 704, Taiwan. ${ }^{5}$ Department of Surgery, Koo Foundation Sun Yat-Sen Cancer Center, Taipei 112, Taiwan. ' ${ }^{6}$ epartment of Radiation Oncology, Koo Foundation Sun Yat-Sen Cancer Center, Taipei 112, Taiwan. ${ }^{7}$ Division of Hematology/Oncology, Department of Internal Medicine, National Cheng Kung University Hospital, Tainan 704, Taiwan. ${ }^{8}$ Graduate Institute of Medicine, College of Medicine, Kaohsiung Medical University, Kaohsiung 807, Taiwan.

\section{Received: 8 April 2019 Accepted: 8 July 2019}

Published online: 19 July 2019

\section{References}

1. Yang $Y$, Bedford MT. Protein arginine methyltransferases and cancer. Nat Rev Cancer. 2013;13(1):37-50. https://doi.org/10.1038/nrc3409.

2. Blanc RS, Richard S. Arginine methylation: the coming of age. Mol Cell. 2017;65(1):8-24. https://doi.org/10.1016/j.molcel.2016.11.003.

3. Frankel A, Clarke S. PRMT3 is a distinct member of the protein arginine $\mathrm{N}$-methyltransferase family. Conferral of substrate specificity by a zinc-finger domain. J Biol Chem. 2000;275(42):32974-82. https://doi.org/10.1074/jbc. M006445200.

4. Tang J, Gary JD, Clarke S, Herschman HR. PRMT 3, a type I protein arginine $\mathrm{N}$-methyltransferase that differs from PRMT1 in its oligomerization, subcellular localization, substrate specificity, and regulation. J Biol Chem. 1998;273(27):16935-45.

5. Bedford MT, Clarke SG. Protein arginine methylation in mammals: who, what, and why. Mol Cell. 2009;33(1):1-13. https://doi.org/10.1016/j.molcel.2 008.12 .013$.

6. Lee J, Sayegh J, Daniel J, Clarke S, Bedford MT. PRMT8, a new membranebound tissue-specific member of the protein arginine methyltransferase family. J Biol Chem. 2005;280(38):32890-6. https://doi.org/10.1074/jbc.M506 944200.

7. Kousaka A, Mori Y, Koyama Y, Taneda T, Miyata S, Tohyama M. The distribution and characterization of endogenous protein arginine $\mathrm{N}$ methyltransferase 8 in mouse CNS. Neuroscience. 2009;163(4):1146-57. https://doi.org/10.1016/j.neuroscience.2009.06.061.

8. Strahl BD, Briggs SD, Brame CJ, Caldwell JA, Koh SS, Ma H, et al. Methylation of histone $\mathrm{H} 4$ at arginine 3 occurs in vivo and is mediated by the nuclear receptor coactivator PRMT1. Curr Biol. 2001;11(12):996-1000.

9. Zika E, Fauquier L, Vandel L, Ting JP. Interplay among coactivator-associated arginine methyltransferase 1, CBP, and CIITA in IFN-gamma-inducible MHC-II gene expression. Proc Natl Acad Sci U S A. 2005;102(45):16321-6. https:// doi.org/10.1073/pnas.0505045102

10. Veland N, Hardikar S, Zhong Y, Gayatri S, Dan J, Strahl BD, et al. The arginine methyltransferase PRMT6 regulates DNA methylation and contributes to global DNA hypomethylation in cancer. Cell Rep. 2017;21(12):3390-7. https://doi.org/10.1016/j.celrep.2017.11.082.
11. Bachand F, Silver PA. PRMT3 is a ribosomal protein methyltransferase that affects the cellular levels of ribosomal subunits. EMBO J. 2004;23(13):2641-50. https://doi.org/10.1038/sj.emboj.7600265.

12. Swiercz R, Person MD, Bedford MT. Ribosomal protein S2 is a substrate for mammalian PRMT3 (protein arginine methyltransferase 3). Biochem J. 2005; 386(Pt 1):85-91. https://doi.org/10.1042/BJ20041466.

13. Swiercz R, Cheng D, Kim D, Bedford MT. Ribosomal protein rpS2 is hypomethylated in PRMT3-deficient mice. J Biol Chem. 2007;282(23):16917-23. https://doi.org/10.1074/jbc.M609778200.

14. Smith JJ, Rucknagel KP, Schierhorn A, Tang J, Nemeth A, Linder M, et al. Unusual sites of arginine methylation in poly(A)-binding protein II and in vitro methylation by protein arginine methyltransferases PRMT1 and PRMT3. J Biol Chem. 1999;274(19):13229-34.

15. Lee J, Bedford MT. PABP1 identified as an arginine methyltransferase substrate using high-density protein arrays. EMBO Rep. 2002;3(3):268-73. https://doi.org/10.1093/embo-reports/kvf052.

16. Zou Y, Webb K, Perna AD, Zhang Q, Clarke S, Wang Y. A mass spectrometric study on the in vitro methylation of HMGA1a and HMGA1b proteins by PRMTs: methylation specificity, the effect of binding to AT-rich duplex DNA and the effect of C-terminal phosphorylation. Biochemistry. 2007:46(26): 7896-906. https://doi.org/10.1021/bi6024897.

17. Fronz K, Otto S, Kolbel K, Kuhn U, Friedrich H, Schierhorn A, et al. Promiscuous modification of the nuclear poly(A)-binding protein by multiple protein-arginine methyltransferases does not affect the aggregation behavior. J Biol Chem. 2008;283(29):20408-20. https://doi.org/1 $0.1074 / j b c . M 802329200$.

18. Cote J, Boisvert FM, Boulanger MC, Bedford MT, Richard S. Sam68 RNA binding protein is an in vivo substrate for protein arginine $\mathrm{N}$ methyltransferase 1. Mol Biol Cell. 2003;14(1):274-87. https://doi.org/10.1 091/mbc.E02-08-0484.

19. Guo H, Wang R, Zheng W, Chen Y, Blum G, Deng H, et al. Profiling substrates of protein arginine $\mathrm{N}$-methyltransferase 3 with S-adenosyl-Lmethionine analogues. ACS Chem Biol. 2014;9(2):476-84. https://doi.org/10.1 021/cb4008259.

20. Hsu JM, Chen CT, Chou CK, Kuo HP, Li LY, Lin CY, et al. Crosstalk between Arg 1175 methylation and Tyr 1173 phosphorylation negatively modulates EGFR-mediated ERK activation. Nat Cell Biol. 2011;13(2):174-81. https://doi. org/10.1038/ncb2158.

21. Su HT, Weng CC, Hsiao PJ, Chen LH, Kuo TL, Chen YW, et al. Stem cell marker nestin is critical for TGF-beta1-mediated tumor progression in pancreatic cancer. Mol Cancer Res. 2013;11(7):768-79. https://doi.org/10.115 8/1541-7786.MCR-12-0511.

22. Sun Y, Ponz-Sarvise M, Chang SS, Chang WC, Chen CH, Hsu JL, et al. Proteasome inhibition enhances the killing effect of BikDD gene therapy. Am J Transl Res. 2015;7(2):319-27.

23. Hsu MC, Hung WC, Yamaguchi H, Lim SO, Liao HW, Tsai CH, et al. Extracellular PKM2 induces cancer proliferation by activating the EGFR signaling pathway. Am J Cancer Res. 2016;6(3):628-38.

24. Chou TC, Talalay P. Quantitative analysis of dose-effect relationships: the combined effects of multiple drugs or enzyme inhibitors. Adv Enzyme Regul. 1984;22:27-55.

25. Uhlen M, Fagerberg L, Hallstrom BM, Lindskog C, Oksvold P, Mardinoglu A, et al. Proteomics. Tissue-based map of the human proteome. Science. 2015; 347(6220):1260419. https://doi.org/10.1126/science.1260419.

26. McBride AE, Cook JT, Stemmler EA, Rutledge KL, McGrath KA, Rubens JA. Arginine methylation of yeast mRNA-binding protein Npl3 directly affects its function, nuclear export, and intranuclear protein interactions. J Biol Chem. 2005;280(35):30888-98. https://doi.org/10.1074/jbc.M505831200.

27. Jenkins JL, Tanner JJ. High-resolution structure of human D-glyceraldehyde3-phosphate dehydrogenase. Acta Crystallogr D Biol Crystallogr. 2006;62(Pt 3):290-301. https://doi.org/10.1107/S09074444905042289.

28. Hang R, Liu C, Ahmad A, Zhang Y, Lu F, Cao X. Arabidopsis protein arginine methyltransferase 3 is required for ribosome biogenesis by affecting precursor ribosomal RNA processing. Proc Natl Acad Sci U S A. 2014;111(45): 16190-5. https://doi.org/10.1073/pnas.1412697111.

29. Kim DI, Park MJ, Lim SK, Park Jl, Yoon KC, Han HJ, et al. PRMT3 regulates hepatic lipogenesis through direct interaction with LXRalpha. Diabetes. 2015;64(1):60-71. https://doi.org/10.2337/db13-1394.

30. Tristan C, Shahani N, Sedlak TW, Sawa A. The diverse functions of GAPDH: views from different subcellular compartments. Cell Signal. 2011;23(2):317-23. https://doi.org/10.1016/j.cellsig.2010.08.003. 
31. Sirover MA. Structural analysis of glyceraldehyde-3-phosphate dehydrogenase functional diversity. Int J Biochem Cell Biol. 2014:57:20-6. https://doi.org/10.1016/j.biocel.2014.09.026.

32. Cho JH, Lee R, Kim E, Choi YE, Choi EJ. PRMT1 negatively regulates activationinduced cell death in macrophages by arginine methylation of GAPDH. Exp Cell Res. 2018;368(1):50-8. https://doi.org/10.1016/j.yexcr.2018.04.012.

33. Zhong XY, Yuan XM, Xu YY, Yin M, Yan WW, Zou SW, et al. CARM1 methylates GAPDH to regulate glucose metabolism and is suppressed in liver cancer. Cell Rep. 2018;24(12):3207-23. https://doi.org/10.1016/j.celrep.2 018.08.066.

34. White MR, Khan MM, Deredge D, Ross CR, Quintyn R, Zucconi BE, et al. A dimer interface mutation in glyceraldehyde-3-phosphate dehydrogenase regulates its binding to AU-rich RNA. J Biol Chem. 2015;290(3):1770-85. https://doi.org/10.1074/jbc.M1 14.618165.

35. Jia J, Arif A, Willard B, Smith JD, Stuehr DJ, Hazen SL, et al. Protection of extraribosomal RPL13a by GAPDH and dysregulation by S-nitrosylation. Mol Cell. 2012;47(4):656-63. https://doi.org/10.1016/..molcel.2012.06.006.

36. Yogalingam G, Hwang S, Ferreira JC, Mochly-Rosen D. Glyceraldehyde-3phosphate dehydrogenase (GAPDH) phosphorylation by protein kinase Cdelta (PKCdelta) inhibits mitochondria elimination by lysosomal-like structures following ischemia and reoxygenation-induced injury. J Biol Chem. 2013;288(26):18947-60. https://doi.org/10.1074/jbc.M113.466870.

37. Kowenz-Leutz E, Pless O, Dittmar G, Knoblich M, Leutz A. Crosstalk between C/EBPbeta phosphorylation, arginine methylation, and SWI/SNF/Mediator implies an indexing transcription factor code. EMBO J. 2010;29(6):1105-15. https://doi.org/10.1038/emboj.2010.3.

38. Tennant DA, Duran RV, Gottlieb E. Targeting metabolic transformation for cancer therapy. Nat Rev Cancer. 2010;10(4):267-77. https://doi.org/10.1038/ nrc2817.

39. Ganapathy-Kanniappan S, Geschwind JF. Tumor glycolysis as a target for cancer therapy: progress and prospects. Mol Cancer. 2013;12:152. https:// doi.org/10.1186/1476-4598-12-152.

40. Zong WX, Rabinowitz JD, White E. Mitochondria and cancer. Mol Cell. 2016; 61(5):667-76. https://doi.org/10.1016/j.molcel.2016.02.011.

41. Viale A, Pettazzoni P, Lyssiotis CA, Ying H, Sanchez N, Marchesini M, et al, Oncogene ablation-resistant pancreatic cancer cells depend on mitochondrial function. Nature. 2014:514(7524):628-32. https://doi.org/10.1 038/nature13611.

42. Kuntz EM, Baquero P, Michie AM, Dunn K, Tardito S, Holyoake TL, et al. Targeting mitochondrial oxidative phosphorylation eradicates therapy-resistant chronic myeloid leukemia stem cells. Nat Med. 2017;23(10):1234-40. https:// doi.org/10.1038/nm.4399

43. LeBleu VS, O'Connell JT, Gonzalez Herrera KN, Wikman H, Pantel K, Haigis MC, et al. PGC-1alpha mediates mitochondrial biogenesis and oxidative phosphorylation in cancer cells to promote metastasis. Nat Cell Biol. 2014; 16(10):992-1003, 1-15. https://doi.org/10.1038/ncb3039.

\section{Publisher's Note}

Springer Nature remains neutral with regard to jurisdictional claims in published maps and institutional affiliations.

Ready to submit your research? Choose BMC and benefit from:

- fast, convenient online submission

- thorough peer review by experienced researchers in your field

- rapid publication on acceptance

- support for research data, including large and complex data types

- gold Open Access which fosters wider collaboration and increased citations

- maximum visibility for your research: over $100 \mathrm{M}$ website views per year

At $\mathrm{BMC}$, research is always in progress.

Learn more biomedcentral.com/submissions 